\title{
Analysis of Serial-Search-Based Code Acquisition in the Multiple-Transmit/ Multiple-Receive-Antenna-Aided DS-CDMA Downlink
}

\author{
SeungHwan Won, Member, IEEE, and Lajos Hanzo, Fellow, IEEE
}

\begin{abstract}
In this paper, we investigate the serial-search-based initial code-acquisition performance of direct-sequence code division multiple access (DS-CDMA) employing multiple transmit/ multiple receive antennas when communicating over uncorrelated Rayleigh channels. We characterize the associated performance trends as a function of the number of antennas. It is demonstrated that, in contrast to our expectation, the achievable correctdetection probability degrades in our typical target operational $E_{\mathrm{c}} / I_{0}$ range as the number of transmit antennas is increased. When maintaining a given total transmit power, our findings suggest that increasing the number of transmit antennas results in the combination of the low-energy noise-contaminated signals of the transmit antennas, which ultimately increases the mean acquisition time (MAT). However, it is extremely undesirable to increase the MAT when the system is capable of attaining its target bit-error-ratio performance at reduced signal-power levels, as a benefit of employing multiple transmit antennas.
\end{abstract}

Index Terms-Code acquisition, direct-sequence code division multiple access (DS-CDMA), multiple transmit/multiple receive antennas (MTMR), serial search.

\section{INTRODUCTION}

$\mathbf{T}$ HE APPLICATION of multiple antennas in the downlink (DL) of wireless systems constitutes an attractive technique of reducing the detrimental effects of time-variant multipath fading environments [1], [2]. In intercell synchronous code-division-multiple-access (CDMA) systems, the mobile station's (MS) receiver must be capable of synchronizing a locally generated pseudonoise (PN) code with the received multiuser signals containing the desired user's PN sequence. Substantial research efforts have been devoted to the design of code-acquisition techniques [3], [4]. However, most of them have been designed for single-input single-output systems [3]-[10]. A variety of serial-search [3], [5]-[7], parallelsearch [3], [4], [8], [11], as well as sequential-estimation [9], [10]-based code-acquisition techniques have been proposed in the literature.

Manuscript received May 10, 2005; revised September 12, 2006 and May 12, 2007. This paper was supported in part by the Ministry of Information and Communication (MIC), Republic of Korea, of the EPSRC, UK, and in part by the EU under the auspices of the Newcom and Phoenix projects. The review of this paper was coordinated by Prof. E. Sourour.

The authors are with the School of Electronics and Computer Science, University of Southampton, SO17 1BJ Southampton, U.K. (e-mail: 1h@ecs. soton.ac.uk).

Digital Object Identifier 10.1109/TVT.2007.905400
The initial processing stage of CDMA systems is the acquisition of the correct timing of the incoming signals received both in the DL and the uplink (UL). Hence, our aim is to minimize the mean acquisition time (MAT), which is proportional to both the correct-detection probability and the time required by the acquisition scheme to notice after the elapse of the code-phaseverification period that a false-locking event occurred and to return to the search mode. In the DL, the uncertainty region (or search-window width) corresponds to the full period of the PN sequence, which tends to be quite wide, for example, $\left(2^{15}-1\right)$-chip intervals in the DL of the intercell synchronous CDMA-2000 system [5], [6]. Hence, the MAT is minimized in the context of serial-search techniques by achieving the best possible correct-detection probability, while maintaining a value of the false-alarm probability and false-locking penalty which is as low as possible. The effect of the aforementioned high-uncertainty region of the DL could be potentially mitigated by testing the correct code-alignment hypotheses using parallel-search techniques. ${ }^{1}$ However, given the wide uncertainty region of the DL, the implementation of the parallelsearch-based hardware often becomes impractical due to its high complexity. On the other hand, in the UL code-acquisition scenario, the MS transmits a short preamble in response to the BS's pilot signal. This preamble is short in order to avoid consuming a high amount of energy. The MS's preamble is then aligned in time-using classic correlation techniques-with the pilot signal transmitted by the base station (BS) after the preceding code acquisition in the DL. Based upon the aforementioned procedure, the BS instructs the MS to advance its transmission instant in order to precompensate the effect of the propagation delay imposed on the code phase. Accordingly, the uncertainty region in the UL corresponds to a fraction of the full period of the $\left(2^{15}-1\right)$-chip PN sequence; for example, it is less than $200 \mathrm{PN}$ chip intervals in the UL of the intercell synchronous CDMA-2000 system [11]. Hence, it is worth emphasizing that in the context of the shorter UL uncertainty period, the MAT is more critically dominated by achieving the best possible correct-detection probability than by attaining the lowest possible false-locking probability, since the effect of

\footnotetext{
${ }^{1}$ In [11], the classic parallel-acquisition arrangement was referred to as the maximum-likelihood technique.
} 
the latter is deweighted by the short duration of the UL uncertainty region [11].

The employment of multiple transmit/multiple receive (MTMR) antennas has been intensely promoted in the DL [1], [2], but ironically, there is a paucity of code-acquisition techniques designed for transmit-diversity-aided systems [12]. To phrase it more explicitly, there are no in-depth studies representing the fundamental characteristics of code-acquisition schemes using transmit diversity, although there have been numerous contributions on receive-diversity-aided systems [11], [13]-[15] in the UL. The capacity of the MTMR-antennaassisted systems designed for maximizing either the achievable multiplexing gain or the diversity gain was quantified in [16]. Numerous studies also quantified how well practical MTMR designs approach these ultimate capacity limits under perfectly synchronized conditions [16], [17], but again, there are no in-depth journal papers on MTMR-aided-acquisition studies. Against this background, in this treatise, we investigate a serialsearch-based technique designed for the MTMR-antenna-aided systems. More explicitly, we quantify both the correct-detection probability, as well as the false-alarm probability, as a function of both the signal-to-interference-plus-noise ratio (SINR) per chip $\left(E_{\mathrm{c}} / I_{0}\right)$ and that of the number of antennas. Additionally, we characterize the MAT, parameterized also by the $E_{\mathrm{c}} / I_{0}$ values and the number of antennas in the text of single-dwell serial search (SDSS) [5].

This paper is organized as follows. Section II describes the system investigated, whereas the correct-detection and falsealarm probabilities are analyzed in the context of uncorrelated Rayleigh channels in Section III. The MAT expressions of the code acquisition designed for MTMR-antenna-aided DS-CDMA systems are introduced in Section IV. In Section V, both our numerical and simulation results are discussed, whereas our conclusions are offered in Section VI.

\section{SySTEM DESCRIPTION}

The transmitted signal at the $n$th chip sampling instant of the $k$ th user at the BS of a multiple-transmit-antenna-aided system can be expressed as

$$
x_{n}(k) a_{n}(k) w_{n, p}(k) \sqrt{\frac{E_{\mathrm{c}}(k)}{P}} h\left(t-n T_{\mathrm{c}}\right) \sqrt{2} \cos \left(2 \pi f_{0} t+\phi_{k}\right)
$$

where $p=1, \ldots, P$ is the number of transmit antennas, $x_{n}(k)$ represents the binary input data sequence assuming values of +1 or $-1, a_{n}(k)$ denotes a common PN sequence having a cellspecific code-phase offset, $w_{n, p}(k)$ identifies the specific Walsh code assigned to the $p$ th transmit antenna, $E_{\mathrm{c}}$ denotes the pilot signal energy per PN code chip, $h(t)$ is the impulse response of the pulse-shaping filter, $T_{\mathrm{c}}$ is the chip duration, $f_{0}$ is the carrier frequency, and $\phi_{k}$ is the carrier phase of the $k$ th user's modulator. The total allocated power is equally shared by the $P$ transmit antennas. The tapped-delay line channel model generates $q$ multipath signals arriving with a time delay of $\tau_{i}$ [6], [7]. Therefore, the signal $r_{k, l}$ at each receive antenna is

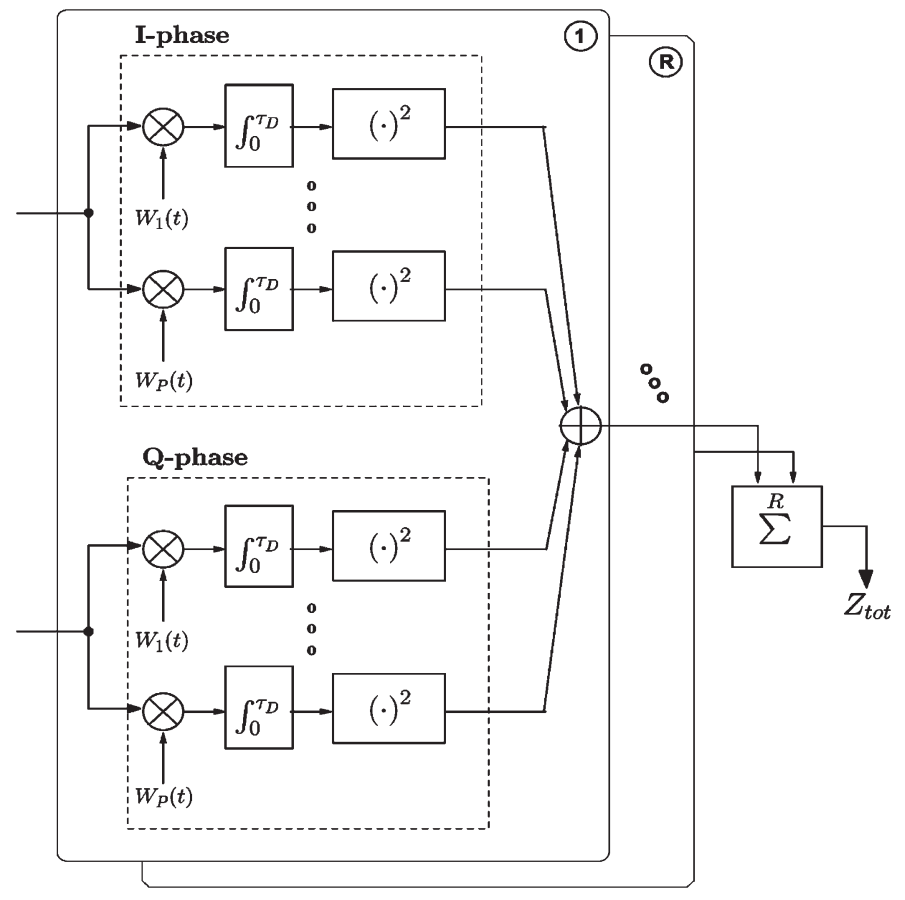

Fig. 1. Receiver structure.

a composite of $q$ multipath signals having a time delay of $\tau_{i}$ expressed in the form of

$$
\begin{aligned}
r_{k, l}=\sum_{i=1}^{q} & \sum_{j=1}^{P} \alpha_{i, j, l} \sqrt{\frac{E_{\mathrm{c}}(k)}{P}} x_{n}(k) a_{n}(k) w_{n, j}(k) \\
& \times h\left(t-n T_{\mathrm{c}}-\tau_{i}\right) \sqrt{2} \cos \left(2 \pi f_{0}^{\prime} t+\phi_{k(i, j, l)}\right)
\end{aligned}
$$

where $l=1, \ldots, R$ is the number of receive antennas, and $\alpha_{i, j, l}$ represents the envelope of the $i$ th path signal obeying the Rayleigh distribution. $f_{0}^{\prime}$ is the carrier frequency distorted by the carrier-frequency mismatch and the Doppler shift. Furthermore, $\phi_{k(i, j, l)}$ denotes the signal phase of the $i$ th path having a uniform distribution over $(0,2 \pi)$. In the case of the initial synchronization of the DS-CDMA, the main goal is to acquire coarse timing of the first arriving path at the receiver, because this timing information is used as that of the reference finger of the Rake receiver. Therefore, we focus our attention on the performance analysis of the first received path and consider the multipath interference effects within the reference cell.

Fig. 1 shows the block diagram of the noncoherent (NC) receiver designed for our code-acquisition scheme using MTMR antennas. As shown in Fig. 1, we generate a decision variable by accumulating $P \cdot R$ independently faded signals observed over a time interval to improve the correct-detection probability in the mobile channel imposing both fading and poor SINR conditions, where the integral dwell time represents $\tau_{D}=N$. $T_{\mathrm{c}}$ and $N$ is defined as $\tau_{D} / T_{\mathrm{c}}$. For the sake of deriving the likelihood function of the decision variable, let us now consider the effects of both timing errors, $\tau$ and frequency mismatches, $\Delta f_{\mathrm{t}}$ on the received signal. The timing errors are imposed by both the delay of the signal received from the mobile channel and the sampling inaccuracy caused by having a finite search step size of $\Delta=T_{\mathrm{c}} / 2$. The total frequency mismatch is the 
sum of the carrier-frequency mismatch $\Delta f_{\mathrm{m}}$ between the BS's transmitter and the MS's receiver, as well as the effect of the Doppler shift $\Delta f_{\mathrm{d}}$.

Following the procedures outlined in [5], the mean values of the $I$ - and $Q$-channel outputs denoted as $E\left[Y_{\text {tot }}^{(I)}(k)\right]$ and $E\left[Y_{\text {tot }}^{(Q)}(k)\right]$ are given by

$$
\begin{aligned}
& \sum_{i=1}^{P} \sum_{j=1}^{R} \alpha_{i, j} \sqrt{\frac{E_{\mathrm{c}}}{P}} R(\tau) \sum_{n=1}^{N} \cos \left[2 \pi n\left(\Delta f_{\mathrm{t}}\right) T_{\mathrm{c}}+\phi_{k(i, j)}\right] \\
& \quad=\sum_{i=1}^{P} \sum_{j=1}^{R} \alpha_{i, j} \sqrt{\frac{E_{\mathrm{c}}}{P}} R(\tau)\left\{A \cdot \cos \phi_{k(i, j)}-B \cdot \sin \phi_{k(i, j)}\right\} \\
& \sum_{i=1}^{P} \sum_{j=1}^{R} \alpha_{i, j} \sqrt{\frac{E_{\mathrm{c}}}{P}} R(\tau) \sum_{n=1}^{N} \sin \left[2 \pi n\left(\Delta f_{\mathrm{t}}\right) T_{\mathrm{c}}+\phi_{k(i, j)}\right] \\
& \quad=\sum_{i=1}^{P} \sum_{j=1}^{R} \alpha_{i, j} \sqrt{\frac{E_{\mathrm{c}}}{P}} R(\tau)\left\{B \cdot \cos \phi_{k(i, j)}+A \cdot \sin \phi_{k(i, j)}\right\}
\end{aligned}
$$

respectively, where the autocorrelation function of the timing error is given by $R(\tau)=\int_{-\infty}^{\infty}\left|H(f)^{2}\right| \cos (2 \pi f \tau) d f$, and the total frequency mismatch is defined as $\Delta f_{\mathrm{t}}=\Delta f_{\mathrm{m}}+$ $\Delta f_{\mathrm{d}}$, as well as $A=\sum_{n=1}^{N} \cos \left[2 \pi n\left(\Delta f_{\mathrm{t}}\right) T_{\mathrm{c}}\right]$, and $B=$ $\sum_{n=1}^{N} \sin \left[2 \pi n\left(\Delta f_{\mathrm{t}}\right) T_{\mathrm{c}}\right]$.

\section{CORRECT-Detection AND \\ FALSE-AlaRM PROBABILITIES}

For the sake of deriving the likelihood functions conditioned on both the hypothesis of the desired signal being present and absent, we introduce the likelihood functions of $Y^{(I)}$ and $Y^{(Q)}$ at each branch, which may be expressed in the context of an additive white Gaussian noise (AWGN) channel [5] as

$$
\begin{aligned}
& p_{0}\left(Y^{(I)}, Y^{(Q)}\right) \\
& =\exp \left[-\left(\left(Y^{(I)}\right)^{2}+\left(Y^{(Q)}\right)^{2}\right) / V\right] / \pi V \\
& p_{1}\left(Y^{(I)}, Y^{(Q)} \mid \phi\right) \\
& =\exp \left[-\left(Y^{(I)}-\sqrt{\frac{E_{\mathrm{c}}}{P}} R(\tau) \cdot C\right)^{2} / V\right] \\
& \quad \times \exp \left[-\left(Y^{(Q)}-\sqrt{\frac{E_{\mathrm{c}}}{P}} R(\tau) \cdot D\right)^{2} / V\right] / \pi V
\end{aligned}
$$

where $A=\sum_{n=1}^{N} \cos \left[2 \pi n\left(\Delta f_{\mathrm{t}}\right) T_{\mathrm{c}}\right]$ and $B=\sum_{n=1}^{N} \sin [2 \pi n$ $\left.\left(\Delta f_{\mathrm{t}}\right) T_{\mathrm{c}}\right], \quad$ as $\quad$ well as $C=A \cos \phi-B \sin \phi$ and $D=$ $B \cos \phi+A \sin \phi$. For simplicity, we omitted the subscripts of $\phi$. The variances of $Y^{(I)}$ and $Y^{(Q)}$ are given by $V_{I}=$ $V_{Q}=V / 2$, where we have $V=N I_{0}$. The total amount of channel-induced impairments in the DL is constituted by the superposition of the background noise, plus the serving-cell interference imposed by the multipath signals and the othercell interference. Further details on the calculation of the total interference may be found in [5] and [6]. Since the carrier phase is modeled by a uniform random variable, we represent the unconditional likelihood function in the presence of the desired signal by averaging (6) over $\phi$, yielding

$$
\begin{aligned}
& p_{1}\left(Y^{(I)}, Y^{(Q)}\right) \\
& =\frac{1}{2 \pi} \int_{0}^{2 \pi} p_{1}\left(Y^{(I)}, Y^{(Q)} \mid \phi\right) d \phi \\
& =\frac{1}{2 \pi} \int_{0}^{2 \pi} \exp \left\{-\left[\left(Y^{(I)}\right)^{2}+\left(Y^{(Q)}\right)^{2}+\frac{N^{2} E_{\mathrm{c}}}{P} R^{2}(\tau)\right.\right. \\
& \left.\left.\times \operatorname{sinc}^{2}\left(N \Delta f_{\mathrm{t}} T_{\mathrm{c}}\right)\right] / V\right\} \\
& \times \exp \left\{2 \sqrt{\frac{E_{\mathrm{c}}}{P}} R(\tau)\right. \\
& \left.\times\left[Y^{(I)} \cdot C+Y^{(Q)} \cdot D\right] / V\right\} \frac{1}{\pi V} d \phi \\
& =\frac{1}{\pi V} \exp \left(-\frac{\left(Y^{(I Q)}\right)^{2}}{V}\right) \\
& \times \exp \left(-\frac{N^{2} E_{\mathrm{c}}}{P} R^{2}(\tau) \operatorname{sinc}^{2}\left(N \Delta f_{\mathrm{t}} T_{\mathrm{c}}\right) / V\right) \\
& \times I_{0}\left(\frac{2 \sqrt{\frac{N^{2} E_{\mathrm{c}}}{P} R^{2}(\tau) \operatorname{sinc}^{2}\left(N \Delta f_{\mathrm{t}} T_{\mathrm{c}}\right)\left(Y^{(I Q)}\right)^{2}}}{V}\right) .
\end{aligned}
$$

where $I_{0}(x) \equiv \int_{0}^{2 \pi} \exp (x \cdot \cos \phi) d \phi / 2 \pi$ is the zeroth-order modified Bessel function, and $\left(Y^{(I Q)}\right)^{2}=\left(Y^{(I)}\right)^{2}+\left(Y^{(Q)}\right)^{2}$. If we take into account a strictly band-limited filter [5], $R(\tau)$ is expressed as

$$
R(\tau)=\frac{\sin \left(\pi \tau / T_{\mathrm{c}}\right)}{\left(\pi \tau / T_{\mathrm{c}}\right)} \equiv \operatorname{sinc}\left(\tau / T_{\mathrm{c}}\right) .
$$

Then, the output variable $Z$ is expressed as

$$
\begin{aligned}
Z & =\left(E\left[Y^{(I)}\right]\right)^{2}+\left(E\left[Y^{(Q)}\right]\right)^{2} \\
& =N^{2}\left(\frac{E_{\mathrm{c}}}{P}\right) \operatorname{sinc}^{2}\left(\frac{\tau}{T_{\mathrm{c}}}\right) \operatorname{sinc}^{2}\left(N \Delta f_{\mathrm{t}} T_{\mathrm{c}}\right)
\end{aligned}
$$

Hence, taking (11) into account, the square of the mean values of $Y^{(I)}$ and $Y^{(Q)}$, which were formulated in (11), is given by

$$
M^{2}=N^{2}\left(\frac{E_{\mathrm{c}}}{P}\right) \operatorname{sinc}^{2}\left(\frac{\tau}{T_{\mathrm{c}}}\right) \operatorname{sinc}^{2}\left(N \Delta f_{\mathrm{t}} T_{\mathrm{c}}\right) .
$$

Thus, the signal-energy reduction expressed as a function of the frequency mismatch becomes

$$
D\left(\Delta f_{\mathrm{t}}\right) \approx \operatorname{sinc}^{2}\left(N \Delta f_{\mathrm{t}} T_{\mathrm{c}}\right)
$$

As a result of the above formulation, the effects of both the timing errors and the frequency mismatches are encapsulated 
by the definition of the squared mean $M^{2}$, which was formulated in (12). This means that the effects of both of these detrimental factors are directly involved in the measured energy value. The other parameters remain the same, as in the case of considering either timing or frequency errors in isolation. By using the Jacobian transformation [18], we arrive at the likelihood functions conditioned on both the hypothesis $p_{0}(Z)$ and $p_{1}(Z)$ of the desired signal being absent and present, respectively, in the context of an AWGN channel as follows:

$$
\begin{aligned}
& p_{0}(Z)=\exp [-Z / V] / V, \quad Z>0 \\
& p_{1}(Z)=\frac{\exp \left[-\left(Z+M^{2}\right) / V\right]}{V} I_{0}\left(\frac{2 \sqrt{M^{2} Z}}{V}\right) .
\end{aligned}
$$

For the sake of expressing the likelihood function conditioned on the presence of the desired signal in $p_{1}(Z)$ derived for transmission over an uncorrelated Rayleigh channel, first, the likelihood function $p_{1}(Z, \beta)$ corresponding to the SINR $\beta$ conditioned on the hypothesis of the desired signal being transmitted over an AWGN channel is weighted by the probability of occurrence $p(\beta)$ of encountering the $\operatorname{SINR} \beta$, as quantified by the likelihood function and then averaged over its range of $-\infty \sim \infty$, yielding

$$
\begin{aligned}
p_{1}(Z)= & \int_{-\infty}^{\infty} p(\beta) p_{1}(Z, \beta) d \beta \\
= & \int_{0}^{\infty}\left(\frac{e^{-\beta / \sigma^{2}}}{\sigma^{2}}\right) \cdot \frac{\exp \left[\left(-Z+\beta M^{2}\right) / V\right]}{V} \\
& \quad \times I_{0}\left(\frac{2 \sqrt{\beta M^{2} Z}}{V}\right) d \beta \\
= & \frac{\exp \left[-Z /\left(V+M^{2} \sigma^{2}\right)\right]}{\left(V+M^{2} \sigma^{2}\right)} \\
\equiv & \frac{\exp \left[-Z /\left(V+\overline{M^{2}}\right)\right]}{\left(V+\overline{M^{2}}\right)}
\end{aligned}
$$

where we have $\overline{M^{2}} \equiv M^{2} \sigma^{2}=N^{2} \overline{E_{\mathrm{c}}}$. Upon defining $V_{\mathrm{F}}=$ $V+\overline{M^{2}}=N\left(I_{0}+N \overline{E_{\mathrm{c}}}\right)$, and $\overline{E_{\mathrm{c}}}=\sigma^{2}\left(E_{\mathrm{c}} / P\right) \operatorname{sinc}^{2}\left(\tau / T_{\mathrm{c}}\right)$ $\operatorname{sinc}^{2}\left(N \Delta f_{\mathrm{t}} T_{\mathrm{c}}\right)$, we arrive at

$$
\begin{aligned}
& p_{0}(Z)=\frac{1}{V} e^{-Z / V} \\
& p_{1}(Z)=\frac{1}{V_{\mathrm{F}}} e^{-Z / V_{\mathrm{F}}} .
\end{aligned}
$$

In this paper, we essentially limit ourselves to considering MTMR antennas without postdetection integration (PDI), noting that PDI is capable of improving the achievable performance upon generating the decision variable by accumulating $L$ consecutive signals observed over multiple time intervals, which improves the correct-detection probability in a mobile channel imposing both the fading and poor SINR conditions [5], [6], [12]. Nonetheless, we will briefly highlight the beneficial effects of additionally using MTMR antennas in conjunction with PDI. Here, we assume that the signals collected in multiple time intervals are independent in both the spatial and the time domains, which leads to a straightforward extension of the formulas to the more general scenarios of invoking MTMR antennas. Since the final decision variable is constituted by the sum of $(P \cdot R \cdot L)$ number of independent variables $\left(Z_{\mathrm{tot}}=\sum_{i=1}^{P} \sum_{j=1}^{R} \sum_{k=1}^{L} Z_{i, j, k}\right)$, each of which has a likelihood function given by (20) or (21), we can determine the Laplace transform of each by raising them to the $(P \cdot R \cdot L)$ th power and then carrying out the inverse transform for the sake of generating the desired likelihood function [5].

Assuming that the number of PDI stages is $L$, in the case of the MTMR antennas using PDI, we arrive at

$$
\begin{aligned}
& p_{0}(Z)=\frac{Z^{P R L-1} e^{-Z / V}}{(P R L-1) ! V^{P R L}} \\
& p_{1}(Z)=\frac{Z^{P R L-1} e^{-Z / V_{\mathrm{F}}}}{(P R L-1) ! V_{\mathrm{F}}^{P R L}} .
\end{aligned}
$$

Using the procedures outlined above, the probability of a correct detection is obtained as follows:

$$
P_{D}=\int_{\theta}^{\infty} p_{1}(Z) d Z=\exp \left(-\frac{\theta}{V_{\mathrm{F}}}\right) \sum_{k=0}^{P R L-1} \frac{\left(\theta / V_{\mathrm{F}}\right)^{k}}{k !} .
$$

Similarly, the probability of false alarm is expressed as

$$
P_{\mathrm{F}}=\int_{\theta}^{\infty} p_{0}(Z) d Z=\exp \left(-\frac{\theta}{V}\right) \sum_{k=0}^{P R L-1} \frac{(\theta / V)^{k}}{k !}
$$

where $\theta$ is a threshold value.

\section{MAT AnAlysis of Code ACQUisition}

The explicit MAT formula derived for a single-antennaassisted serial-search-based code-acquisition arrangement were provided in [5]. There is no difference between a singleantenna- and a multiple-antenna-aided DS-CDMA system in terms of characterizing their MAT performance, except for the derivation of the correct-detection and the false-alarm probabilities as a function of both the number of MTMR antennas. We will continue our discourse here by characterizing the MAT performance of the NC scheme employing SDSS [5]. We postulate that in each chip duration $T_{\mathrm{c}}, l$ number of correcttiming hypotheses are examined, which are spaced by $T_{\mathrm{c}} / l$. Hence, the number of intervals in the uncertainty region is increased $l$-fold. All the resultant $(\nu-2 l)$ number of the states that may lead to a false alarm are expected to increase the MAT proportionately to the corresponding penalty time. The $2 l$ legitimate locking states within a lag of one chip duration of the correct-timing instant are taken into account in the 
MAT analysis. Combining all these $2 l$ legitimate locking states into the correct-detection transfer function, encompassing all the branches of the relevant state diagram in [5, Fig. 3.4], leads to the correct-detection transfer function, which is expressed as

$$
H_{D}(z)=\sum_{j=1}^{2 l} P_{D j} z \prod_{i=1}^{j-1}\left[\left(1-P_{D i}\right) z\right]
$$

where $P_{D j}$ represents the correct-detection probability associated with the $j$ th correct detection, following $(j-1)$ trials, each resulting in a miss and where each probability of a miss in the corresponding total miss transfer function is given by $\left(1-P_{D i}\right)$. Furthermore, $z$ indicates the unit-delay operator, and the exponent of $z$ represents the time delay, whereas $H_{0}(z)$ in [5, Fig. 3.3] denotes the absence of the desired user's signal at the output of the acquisition scheme, which is expressed as

$$
H_{0}(z)=\left(1-P_{\mathrm{F}}\right) z+P_{\mathrm{F}} z^{K+1}
$$

where $P_{\mathrm{F}}$ represents the false-alarm probability, and $K$ is the false-locking-penalty factor [5]. Finally, $H_{\mathrm{M}}(z)$ represents the overall miss probability of a search run carried out across the entire uncertainty region, which may be formulated as the product of the individual miss probabilities, since these may be considered independent events, yielding

$$
H_{M}(z)=z^{2 l} \prod_{j=1}^{2 l}\left(1-P_{D j}\right)
$$

Then, it may be shown that the generalized expression formulated for calculating the MAT of the serial-search-based codeacquisition scheme is given by [5]

$$
\begin{aligned}
& E\left[T_{A C Q}\right]=\frac{1}{H_{D}(1)}\left[H_{D}^{\prime}(1)+H_{M}^{\prime}(1)\right. \\
& \left.+\left\{(\nu-2 l)\left[1-\frac{H_{D}(1)}{2}\right]+\frac{1}{2} H_{D}(1)\right\} H_{0}^{\prime}(1)\right] \cdot \tau_{D}
\end{aligned}
$$

where $\left.H_{x}^{\prime}(z)\right|_{x=D, M \text {, or } 0}$ represents a derivative of

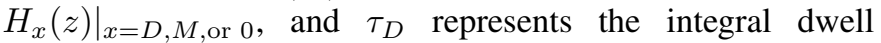
time. In the following section, we will evaluate the achievable system performance based on both the numerical analysis and the simulations.

\section{Performance Analysis}

In this section, we will characterize the code-acquisition performance of the MTMR-antenna-aided CDMA systems. The system parameters are summarized in Table I. In Table II, we outlined the maximum SINR degradation imposed by both

\begin{tabular}{|c|c|c|}
\hline \multicolumn{2}{|l|}{ Bandwidth } & $1.2288 \mathrm{MHz}$ \\
\hline \multicolumn{2}{|c|}{ Carrier frequency } & $1.9 \mathrm{GHz}$ \\
\hline \multicolumn{2}{|c|}{ Spreading factor } & 128 \\
\hline \multirow{2}{*}{ Diversity: } & Transmit & $1,2,4,6,8,10$ \\
\hline & Receive & $1,2,4$ \\
\hline \multicolumn{2}{|c|}{ Frequency mismatch } & $1000 \mathrm{~Hz}$ \\
\hline \multicolumn{2}{|c|}{ Mobile speed } & $160 \mathrm{~km} / \mathrm{h}$ \\
\hline \multicolumn{2}{|c|}{ Coherent integration interval } & 256chips \\
\hline \multicolumn{2}{|c|}{ Total uncertainty region } & 65534 \\
\hline \multicolumn{2}{|c|}{ False locking penalty factor } & 1000chips \\
\hline
\end{tabular}
the Doppler shift and the frequency mismatch between the transmitter and the receiver in conjunction with a coherent
TABLE I

SYSTEM PARAMETERS

TABLE II

MAXIMUM SINR DEGRADATION INFLICTED BY BOTH THE DOPPLER SHIFT AND A 1000-Hz FREQUENCY MismatCH IN CONJUNCTION WITH THE COHERENT INTEGRATION INTERVAL OF $N$ CHIP DURATIONS AT A CARRIER FREQUENCY OF $1.9 \mathrm{GHz}$

\begin{tabular}{|l|l|l|l|l|l|}
\hline N (Chips) & 64 & 128 & 256 & 384 & 512 \\
\hline Degradation (dB) & 0.061 & 0.245 & 0.997 & 2.314 & 4.321 \\
\hline
\end{tabular}

integration interval of $N$ chip durations. The length of the PN sequence in our system was assumed to be $2^{15}-1(65534) \cdot T_{\mathrm{c}}$, where the chip duration is $T_{\mathrm{c}}=1 / 1.2288 \mu \mathrm{s}$. It may be deemed sufficient at this point to integrate the detector output shown in Fig. 1 over $N=256$ chips, which is equivalent to two 128-chip modulated symbols used for coherent accumulation. This value was calculated by using (13) provided for determining the performance degradation owing to both the Doppler shift and the frequency mismatch. The spreading factor of the Walsh code to be acquired was selected to be 128 . The frequency mismatch was assumed to be $1000 \mathrm{~Hz}$ [5], whereas the carrier frequency was $1.9 \mathrm{GHz}$. As a worst case mobile speed, it is reasonable to postulate $160 \mathrm{~km} / \mathrm{h}$. We also assumed that the sampling inaccuracy caused by having a finite search step size of $\Delta=T_{\mathrm{c}} / 2$ was $-0.91 \mathrm{~dB}$, which is a typical value of the search step size [5], [6]. Accordingly, we considered three performance-degradation factors, which encompassed the frequency mismatch, the Doppler shift, and the effects of the finite sampling distance. All these imperfections were taken into account when calculating the correct-detection probability. Finally, two additional parameters have to be stipulated for the analysis of the SDSS. Specifically, the total uncertainty region was assumed to entail 65534 hypotheses, and in the spirit of [6], the false-locking-penalty factor was assumed to be 1000 chip durations.

Fig. 2 shows the correct-detection probability versus the false-alarm probability, parameterized by both the number of transmit antennas for $P=1,2$, as well as 4 , and the $E_{\mathrm{c}} / I_{0}$ value. In the case of $E_{\mathrm{c}} / I_{0}=-10 \mathrm{~dB}$, the achievable performance enhancement gradually saturates as the transmit diversity order is increased from $P=1$ to 4 . By contrast, $P_{D}$ decreases as the number of transmit antennas $P$ increases, when the MS experiences a relatively low $E_{\mathrm{c}} / I_{0}$ value of $-19 \mathrm{~dB}$, as evidenced by the three curves corresponding to lower $P_{D}$ values in Fig. 2. Figs. 3 and 4 characterize the correct-detection probability versus the false-alarm probability, parameterized by both the number of transmit antennas for $P=1,2$, as well as 4 , in conjunction with both $R=2$ (Fig. 3) 


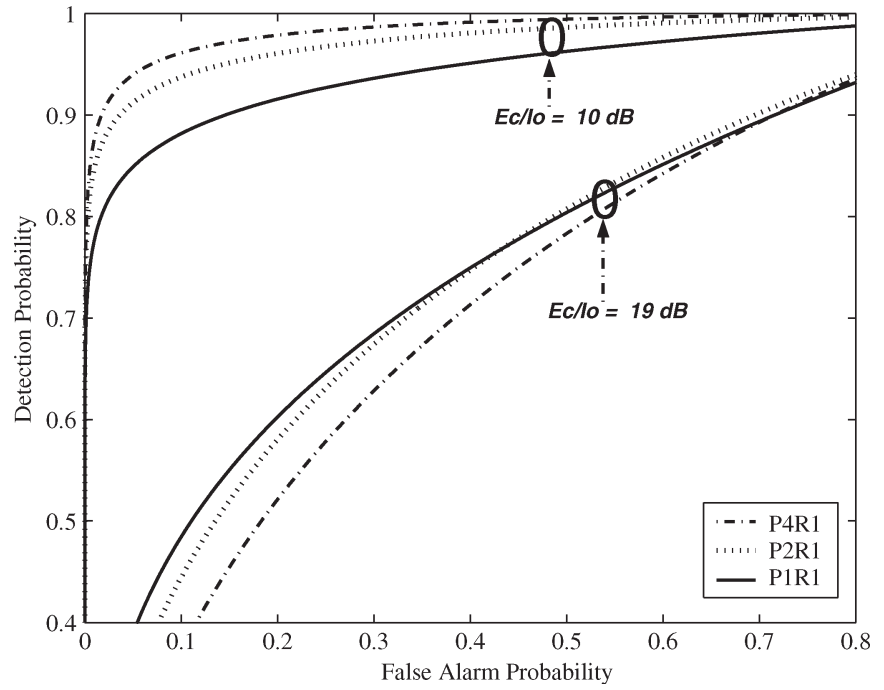

Fig. 2. Correct-detection probability versus false-alarm probability for $P=1,2$, and 4 transmit antennas in conjunction with $R=1$ receive antenna.

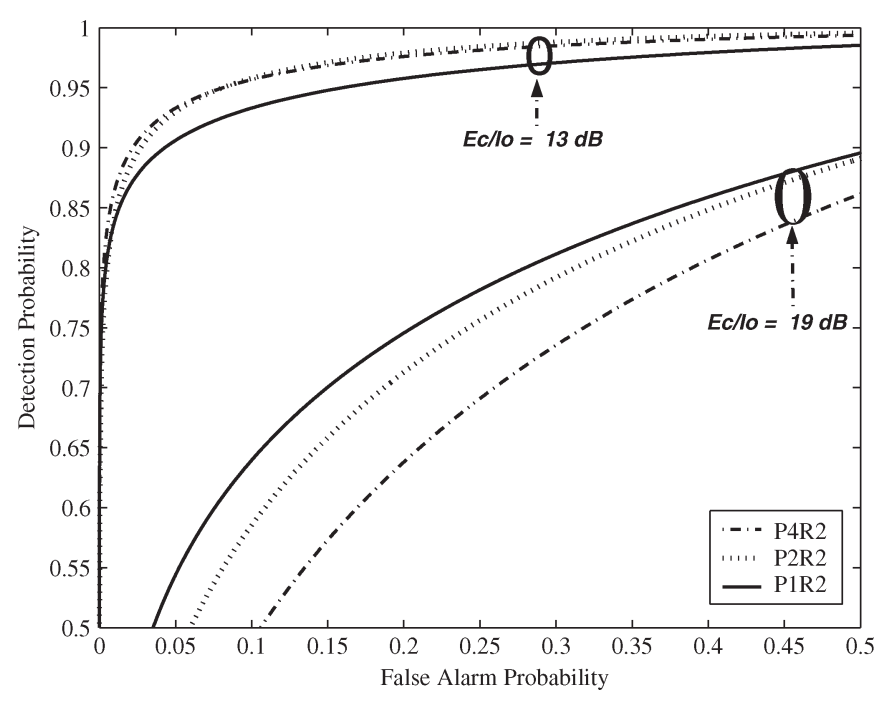

Fig. 3. Correct-detection probability versus false-alarm probability for $P=1,2$, and 4 transmit antennas in conjunction with $R=2$ receive antennas.

and $R=4$ (Fig. 4) receive antennas and as a function of the $E_{\mathrm{c}} / I_{0}$ value.

When having $R=2$ receive antennas, as shown in Fig. 3, the results show similar trends to those of Fig. 2. By contrast, in the scenario of $R=4$ receive antennas as shown in Fig. 4, there is a sufficiently high spatial diversity gain, which has beneficial effects on the achievable acquisition performance $P_{D}$. However, as shown in Fig. 4 , increasing the transmit diversity order imposes a degradation of the achievable $P_{D}$ performance. The specific $E_{\mathrm{c}} / I_{0}$ abscissa values in Figs. 2-4 were chosen to exemplify the typical achievable values. In all the remaining figures, we will assume an operation in the range of "finger locking," which may be considered to be the range between $E_{\mathrm{c}} / I_{0}=-17$ and $-13 \mathrm{~dB}$, as suggested in [19] and [20]. Therefore, in Figs. 5 and 6, we will investigate in more detail the somewhat unexpected phenomenon of experiencing a degraded acquisition performance in the presence of multiple transmit antennas. The correct-detection probabilities shown in

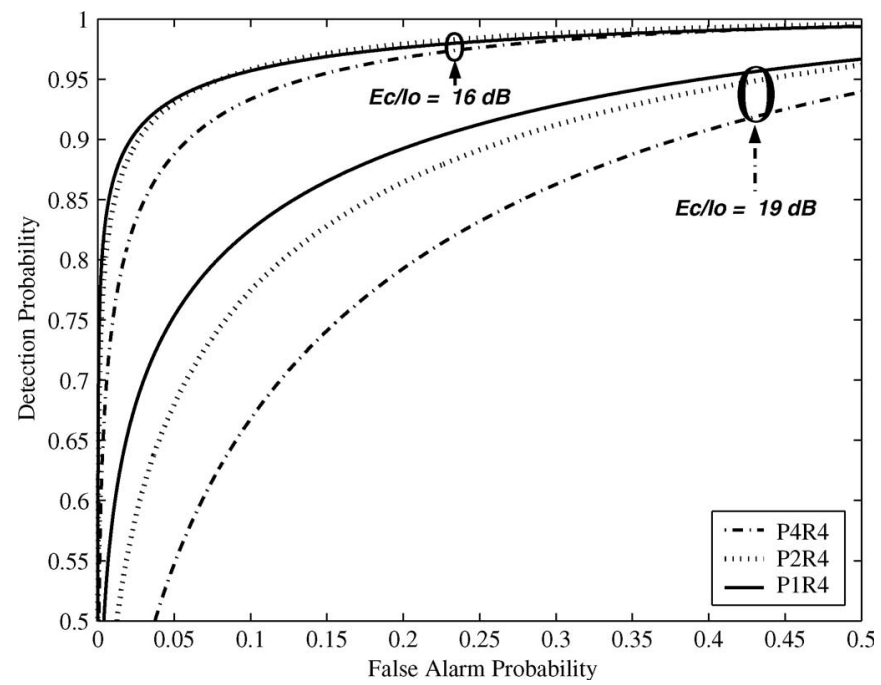

Fig. 4. Correct-detection probability versus false-alarm probability for $P=1,2$, and 4 transmit antennas in conjunction with $R=4$ receive antennas.

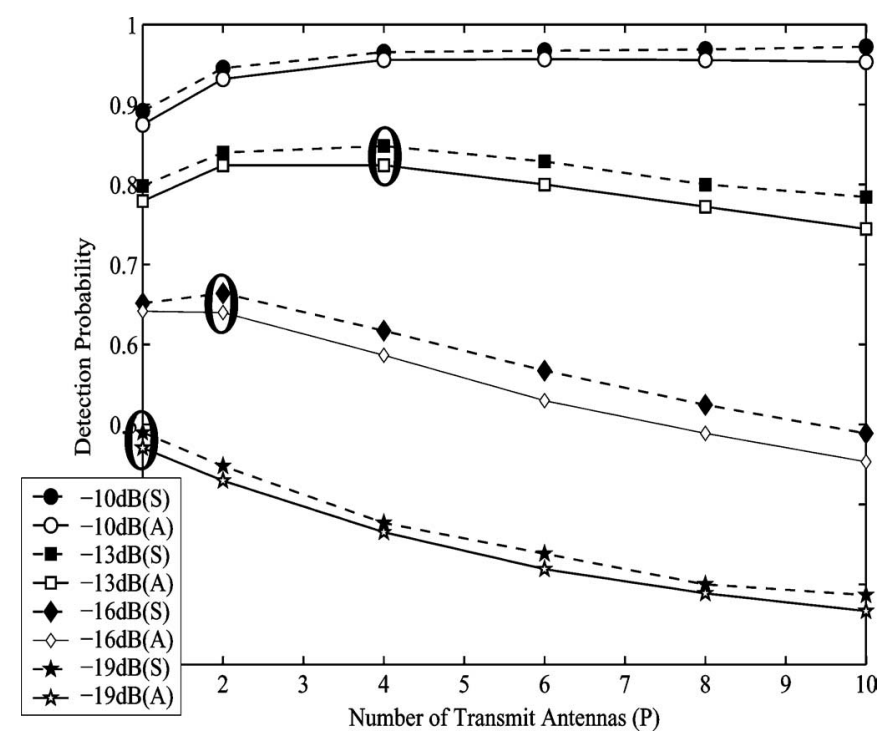

Fig. 5. Correct-detection probability versus the number of transmit antennas for $P=1,2,4,6,8$, and 10 , parameterized by the pilot channel's $E_{c} / I_{0}$ value.

Figs. 5 and 6 were obtained assuming a false-locking probability of $P_{\mathrm{F}}=0.1$ for all the scenarios considered, where ' $S$ ' denotes the simulation results, whereas ' $A$ ' presents the numerical analysis results calculated from both (24) and (25). The simulations always represent a slightly better correct-detection probability than the analysis at the same false-alarm probability. This is because, in the analysis, a constant Doppler-shift value calculated for the worst-case scenario was added to the total frequency mismatch in (13). However, in a practical scenario, the Doppler shift has either a positive or a negative impact on the $P_{D}$ performance, depending upon the specific conditions encountered.

In Figs. 5 and 6, the relationship between $P_{D}$ and the number of transmit antennas is portrayed both with and without multiple receive antennas for different values of $E_{\mathrm{c}} / I_{0}$, respectively. More explicitly, Fig. 5 shows the correct-detection probability versus the number of transmit antennas, parameterized by the 


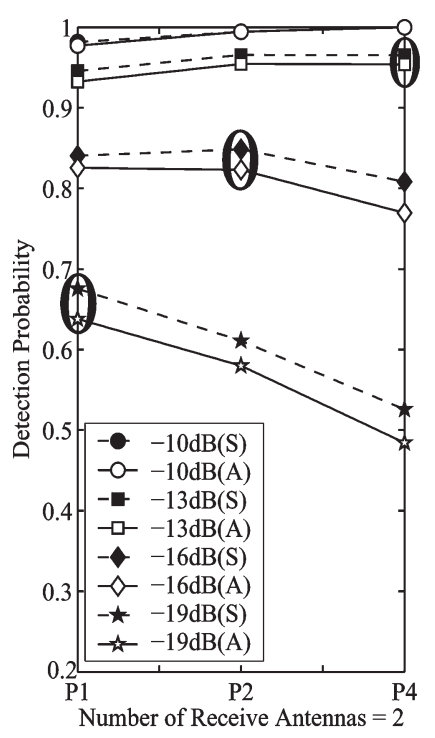

(a)

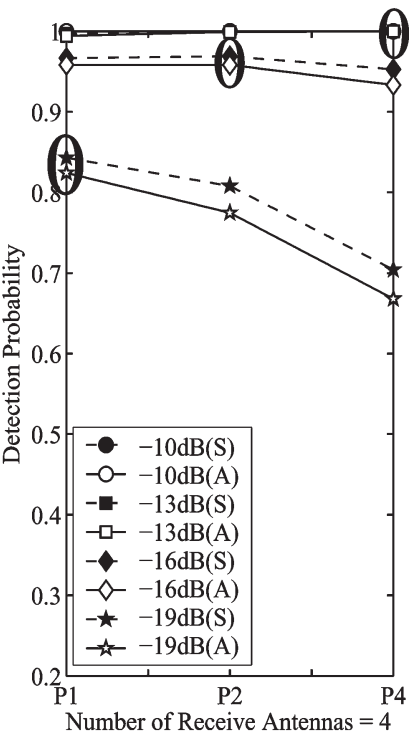

(b)
Fig. 6. Correct-detection probability versus the number of transmit antennas for $P=1,2$, as well as 4 , and the number of receive antennas for both (left) $R=2$ and (right) $R=4$, parameterized by the pilot channel's $E_{c} / I_{0}$ value.

pilot channel's $E_{\mathrm{c}} / I_{0}$ value. At $E_{\mathrm{c}} / I_{0}=-10 \mathrm{~dB}$, a slight $P_{D}$ improvement is observed upon increasing the number of transmit antennas, although, again, at typically low $E_{\mathrm{c}} / I_{0}$ values, the opposite is true. Fig. 6 characterizes the correctdetection probability versus both the number of MTMR antennas, parameterized by the pilot channel's $E_{\mathrm{c}} / I_{0}$ value. The left illustration of Fig. 6 characterizes the scenario of $R=2$ receive antennas, whereas the one at the right was valid for $R=4$ receive antennas. The curve recorded for $E_{\mathrm{c}} / I_{0}=-10 \mathrm{~dB}$ at the right of Fig. 6 overlapped with that plotted for $E_{\mathrm{c}} / I_{0}=$ $-13 \mathrm{~dB}$ because all the achievable detection probabilities were $P_{D}=1$. Both Figs. 5 and 6 show that $P_{D}$ tends to decrease as the number of transmit antennas increases, particularly when the MS experiences a low $E_{\mathrm{c}} / I_{0}$ value. We can observe in both Figs. 5 and 6 that the highest detection probabilities marked by circles were achieved, when the per-branch $E_{\mathrm{c}} / I_{0}$ value was $-19 \mathrm{~dB}$ for a given total $E_{\mathrm{c}} / I_{0}$ value in the range of "finger locking." Owing to the aforementioned facts, the range of the minimum $E_{\mathrm{c}} / I_{0}$ values required for reaching "finger locking" may vary, depending upon the number of transmit antennas.

Let us now proceed by defining the MAT gain as the quotient of the MAT achieved by a particular MTMR-antenna configuration and that attained by the conventional $P 1 R 1$ scheme. However, the transmit power reduction imposed by using multiple transmit antennas can be partially compensated for with the aid of multiple receive antennas. In this case, it is more appropriate to use the MAT of the $P 1 R 2$ or $P 1 R 4$ scheme. Fig. 7 characterizes the MAT gain/degradation as a function of the $E_{\mathrm{c}} / I_{0}$ values considered. Here, the MAT gain/degradation recorded for the different scenarios was presented by defining the MAT ratios of the scenarios $(P 1 R 1 / P x R 1),(P 1 R 2 / P x R 2)$, and $(P 1 R 4 / P x R 4)$, where we have $x=2$ or 4 . To elaborate a little further, there would have been some benefit in using the same normalization factor, such as the MAT of the single-receiver system $R 1$ for all the different scenarios, but we found that

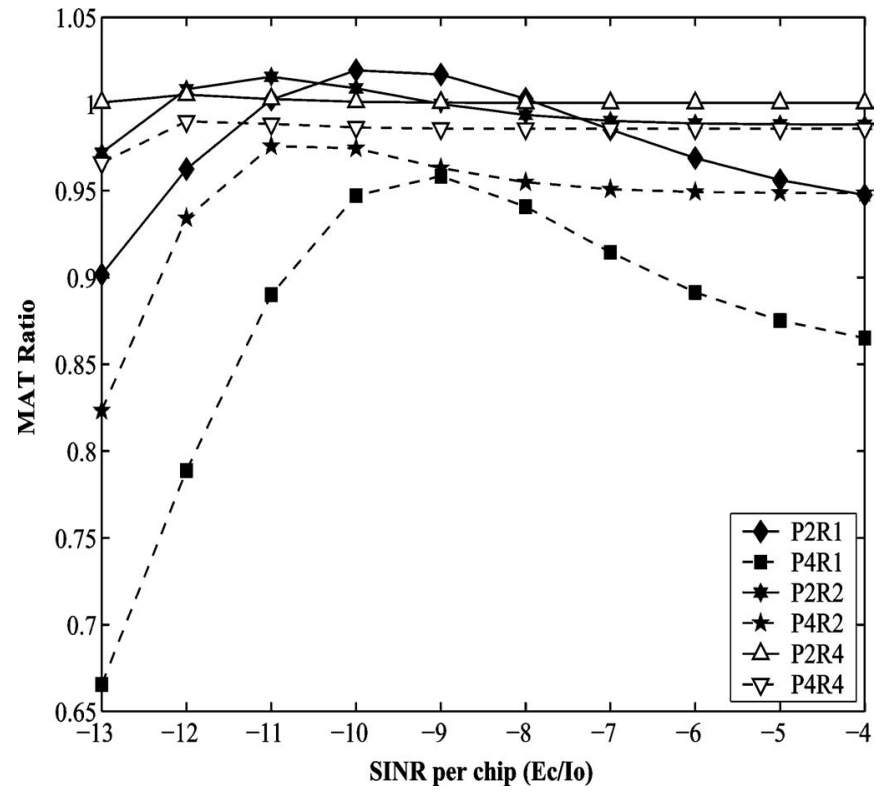

Fig. 7. MAT ratio versus $E_{c} / I_{0}$ value.

the aforementioned definitions were more suitable for explicitly demonstrating the impact of the number of transmit antennas. As shown in Fig. 7, for example, the $P 2 R 1$ and $P 2 R 2$ scenarios exhibit a modest MAT gain for $E_{\mathrm{c}} / I_{0}$ values between -11 and $-8 \mathrm{~dB}$ and between -12 and $-9 \mathrm{~dB}$, respectively, but in the rest of the SINR region, a MAT degradation is experienced. Here, all the performance curves have been generated at the threshold value of $E_{\mathrm{c}} / I_{0}=-13 \mathrm{~dB}$, which was considered as the minimum value required for reliable finger locking. More quantatively, observe in Fig. 7 that in order to achieve the MAT ratio of unity, corresponding to no transmit-antenna-induced MAT loss, we need about $2 \mathrm{~dB}$ more power for the $P 2 R 1$ scenario. Alternatively, the cell radius would have to be reduced by an appropriate path-loss-dependent factor, if no transmitantenna-induced MAT degradation can be tolerated. Therefore, the employment of MTMR antennas may lead to the reduction of the attainable cell size, since no communications are possible until code acquisition has been completed.

In summary, multiple transmit antennas constitute an efficient means of improving the attainable diversity gain, and/or system throughput, when communicating over mobile channels. However, for the sake of fair comparisons, the total signal power transmitted from the multiple transmit antennas must be fixed, regardless of their number. In other words, the total transmit power must be equally shared by all the transmit antennas. This implies that an excessively low level of per branch received signal strength would lead to a low acquisition performance, even if the transmit multiplexing/diversity gain is high. In other words, a high-diversity order effectively results in an acquisition performance loss, as a consequence of the insufficiently high transmit signal strength per branch. In the case of employing both multiple transmit and receive antennas, this trend is still observable, although using two or four receive antennas has the potential of mitigating the associated acquisition performance degradation imposed by the low per branch $E_{\mathrm{c}} / I_{0}$ values encountered. 


\section{CONCLUSION}

In this paper, we analyzed the MTMR-antenna-aided initial acquisition performance of the intercell synchronous CDMA DL. The probabilities of a correct detection and a false alarm have been derived analytically, and both numerical and simulation results were provided. Ironically, our findings suggest that increasing the number of transmit antennas results in combining the low-energy noise-contaminated signals of the transmit antennas, which ultimately reduces the correctdetection probability. However, it is extremely undesirable to degrade the achievable acquisition performance, when the system is capable of attaining its target bit-error-rate performance at reduced SINR values, as a benefit of employing multiple transmit antennas. It may be concluded that the achievable cell coverage determined by the received pilot channel power may be reduced as the number of transmit antennas is increased, which is a highly undesirable phenomenon since it has grave repercussions in terms of having to tolerate a high number of handovers per cell. Our future research will focus on the study of iterative turbo-like acquisition schemes [4], [10] specifically designed for multiple-input multiple-output systems.

\section{REFERENCES}

[1] D. Gesbert, M. Shafi, D. S. Shiu, P. J. Smith, and A. Naguib, "From theory to practice: An overview of MIMO space-time coded wireless systems," IEEE J. Sel. Areas Commun., vol. 21, no. 3, pp. 281-302, Apr. 2003.

[2] R. A. Soni and R. M. Buehrer, "On the performance of open-loop transmit diversity techniques for IS-2000 systems: A comparative study," IEEE Trans. Wireless Commun., vol. 3, no. 5, pp. 1602-1615, Sep. 2004.

[3] B.-G. Lee and B.-H. Kim, Scrambling Techniques for CDMA Communications. Norwell, MA: Kluwer, 2001, ch. 2 and 3.

[4] L. Hanzo, L.-L. Yang, E.-L. Kuan, and K. Yen, "Single- and multicarrier DS-CDMA, IV multi-carrier CDMA," in Initial Synchronization of DS-CDMA and MC-CDMA Systems. Hoboken, NJ: Wiley, 2003 , ch. 21.

[5] A. J. Viterbi, CDMA: Principles of Spread Spectrum Communication. Reading, MA: Addison-Wesley, 1995, ch. 2 and 3.

[6] H. R. Park, "Performance analysis of a double-dwell serial search technique for cellular CDMA networks in the case of multiple pilot signals," IEEE Trans. Veh. Technol., vol. 48, no. 6, pp. 1819-1830, Nov. 1999.

[7] L.-L. Yang and L. Hanzo, "Serial acquisition of DS-CDMA signals in multipath fading mobile channels," IEEE Trans. Veh. Technol., vol. 50, no. 2, pp. 617-628, Mar. 2001.

[8] K. K. Chawla and D. V. Sarwate, "Parallel acquisition of PN sequences in DS/SS systems," IEEE Trans. Commun., vol. 42, no. 5, pp. 2155-2164, May 1994.

[9] R. B. Ward, "Acquisition of pseudonoise signals by sequential estimation," IEEE Trans. Commun. Technol., vol. COM-13, no. 4, pp. 475-483, Dec. 1965.

[10] L.-L. Yang and L. Hanzo, "Acquisition of m-sequences using recursive soft sequential estimation," IEEE Trans. Commun., vol. 52, no. 2, pp. 199 204, Feb. 2004.

[11] H. R. Park and B. J. Kang, "On the performance of a maximumlikelihood code-acquisition technique for preamble search in a CDMA reverse link," IEEE Trans. Veh. Technol., vol. 47, no. 1, pp. 65-74, Feb. 1998.

[12] S. H. Won and Y. J. Kim, "Performance analysis of multi-path searcher for mobile station in W-CDMA system employing transmit diversity," Electron. Lett., vol. 39, no. 1, pp. 137-139, Jan. 2003.

[13] R. R. Rick and L. B. Milstein, "Parallel acquisition of spread-spectrum signals with antenna diversity," IEEE Trans. Commun., vol. 45, no. 8, pp. 903-905, Aug. 1997.

[14] W. H. Ryu, M. K. Park, and S. K. Oh, "Code acquisition schemes using antenna arrays for DS-SS systems and their performance in spatially correlated fading channels," IEEE Trans. Commun., vol. 50, no. 8, pp. 13371347, Aug. 2002.
[15] S. Kim, "Acquisition performance of CDMA systems with multiple antennas," IEEE Trans. Veh. Technol., vol. 53, no. 5, pp. 1341-1353, Sep. 2004.

[16] S. X. Ng and L. Hanzo, "On the MIMO channel capacity of multidimensional signal sets," IEEE Trans. Veh. Technol., vol. 55, no. 2, pp. 528-536, Mar. 2006.

[17] A. Goldsmith, S. A. Jafar, N. Jindal, and S. Vishwanath, "Capacity limits of MIMO channels," IEEE J. Sel. Areas Commun., vol. 21, no. 5, pp. 684 702, Jun. 2003.

[18] A. Leon-Garcia, "Probability and random processes for electrical engineering," in Multiple Random Variables, 2nd ed. Reading, MA Addison-Wesley, 1994, ch. 4.

[19] C. Noblet, M. Fadridis, and R. Owen, "Downlink transmit power issues in a WCDMA cellular system," in Proc. $3 G$ Mobile Commun. Technol., (Conf. Publ. No. 489), May 8-10, 2002, pp. 244-249.

[20] J. S. Lee and L. E. Miller, "CDMA systems engineering handbook," in CDMA Optimization Issues. Norwood, MA: Artech House, 1998, ch. 11.

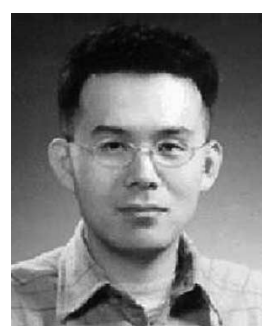

SeungHwan Won (M'04) received the B.S. and M.S. degrees in radio science and engineering from Korea University, Seoul, Korea, in 1999 and 2001, respectively. Since October 2004, he has been working toward the Ph.D. degree with the Communications Research Group, School of Electronics and Computer Science, University of Southampton, Southampton, U.K.

$\mathrm{He}$ was a Research Engineer with the Mobile Communication Technology Research Laboratory, LG Electronics R\&D, from January 2001 to September 2004. His major research interests include initial synchronization in noncoherent multiple-input multiple-output (MIMO)-aided single- and multiple-carrier code division multiple access, interleave division multiple access, and orthogonal frequency division multiple access, as well as in iterative synchronization schemes designed for MIMO-aided single- and multiplecarrier transmission systems.

Dr. Won was the recipient of the 2004 state scholarship of the Information and Telecommunication National Scholarship Program, Ministry of Information and Communication, Korea.

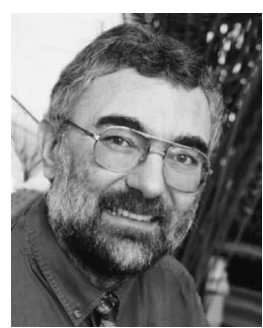

Lajos Hanzo (M'91-SM'92-F'04) received the M.S. degree in electronics and the Ph.D. degree in 1976 and 1983, respectively. He received the D.Sc. degree from the University of Southampton, Southampton, U.K., in 2004

During his career in telecommunications, he has held various research and academic posts in Hungary, Germany, and the U.K. Since 1986, he has been with the School of Electronics and Computer Science, University of Southampton, where he holds the chair in telecommunications. He coauthored 15 John Wiley and IEEE Press books totaling 10000 pages on mobile radio communications, published about 700 research papers, organized and chaired conferences, presented various keynote and overview lectures, and has been awarded a number of distinctions. Currently, he heads an academic research team, working on a range of research projects in the field of wireless multimedia communications sponsored by industry, the Engineering and Physical Sciences Research Council, U.K., the European Information Society Technologies Programme, and the Mobile Virtual Centre of Excellence, Basingstoke, U.K. He is an enthusiastic supporter of industrial-academic liaison. He also offers a range of industrial courses.

Dr. Hanzo is an IEEE Distinguished Lecturer of both the Communications Society and the Vehicular Technology Society. He is a Fellow of the Royal Academy of Engineering and of the IEE. He is a Governor of the IEEE Vehicular TeChNOLOGY Society and an Editor of the ProceEdings of THE IEEE. 